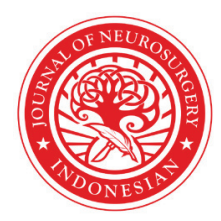

\title{
"Unlocking the brain" - key concepts to accessing the anterolateral skull base made simple
}

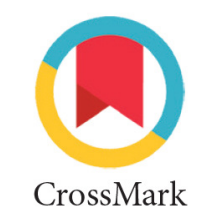

\author{
lype Cherian ${ }^{1 *}$, Hira Burhan ${ }^{1}$
}

\section{ABSTRACT}

Background: The close relationships of skull base structures make it crucial to approach it in a fashion that provides wider exposure with minimal retraction. The concept of brain unlocking adds to the axial and sagittal views allowing access to the optico-carotid window with much ease as compared to the only sylvian dissection in an oblique plane. Another concept introduced is a modification of the Dolenc approach that prevents the bleeding from the cavernous sinuses and also protects the spanning nerves from paresis while operating within the cavernous sinus.

Methods: Unlocking the frontal and temporal lobes in sagittal and axial planes involves drilling the sphenoid bone and sharply dissecting the orbito-meningeal band, respectively. The peeling of the temporal lobe from the cavernous sinus is carried out while keeping the true cavernous membrane intact. This is a modification of the Dolenc approach.

Results: The outcomes of these maneuvers is clearly depicted in the cadaver and surgical dissections performed for skull base approaches. The immediate effects of brain unlocking can be seen as the brain flattens out completely at the fronto-temporal junction. This, followed by a modified Dolenc maintaining a pericavernous plane helps reduce the bleeding considerably.

Conclusion: The use of brain unlocking maneuvers can be essential in providing a wider surgical corridor to approach deep structures in the middle fossa. Maintaining a pericavernous plane while peeling the temporal dura can help reduce cavernous bleeding and prevent nerve paresis in the region.

Keywords: anterolateral skull base; sphenoid; orbito-meningeal band; Dolenc; cavernous sinus; anterior clinoid; petrosectomy Cite This Article: Cherian, I., Burhan, H. 2020. “Unlocking the brain” - key concepts to accessing the anterolateral skull base made simple. Indonesian Journal of Neurosurgery 3(3): 96-100. D0I: 10.15562/ijn.v3i3.103

${ }^{1}$ Nobel Institute of Neurosciences, Nobel Medical College and Teaching Hospital, Biratnagar, Nepal
*Corresponding to: lype Cherian; Nobel Institute of Neurosciences, Nobel Medical College and Teaching Hospital, Biratnagar, Nepal; drrajucherian@gmail.com
Received: 2020-01-14

Accepted: 2020-08-27 Published: 2020-11-27

\section{INTRODUCTION}

The skull base surgery has its origins from the fact that "bone drilling in the base decreases the need for or avoids brain retraction". However, this drilling is done primarily to access areas in the base of the brain like the suprasellar cisterns, optic nerves, carotids and the circle of Willis, the interpeduncular fossa and so on. It is therefore, these structures which are of interest to a skull base surgeon.

Considering the complex and intricate anatomy of the skull base, it is very important to have an exposure that allows an operating corridor for the surgeon. The brain is like a tube which is folded in 3-dimensions: sagittal, axial and oblique planes. The concept of brain "unlocking" is crucial for micro-neurosurgical procedures which can be performed in all the three planes. A pterional approach with sylvian dissection, is the most widely used and explained in depth over the years in micro-neurosurgery. This approach helps in unfolding the brain to a certain extent, in an oblique fashion, however, limiting the surgeon to only one surgical window which serves insufficient in a tight traumatic brain. Therefore, extradural maneuvers to "unlock" the fronto-temporal folds of the brain may be used to get to the base of the brain with a wider exposure.

In this article we describe the concept of brain unlocking in a multiplanar view, and explain in detail, a modification of the Dolenc approach to reach the anterolateral skull base in a bloodless fashion.

\section{Brain "unlocking" - the anatomical concept}

The frontal and temporal lobes are "unlocked" from each other in the sagittal and axial fashion by drilling off the sphenoid and dissecting the Orbitomeningeal band, respectively. These maneuvers are performed extra-durally and open the tight dural curves, ultimately resulting in a flattened brain surface as shown below (Figure 1).

Once the dura is exposed, the sphenoid ridge and the temporal bone is drilled as much as possible to unfold the frontal lobe from the temporal lobe in a sagittal manner, providing a major view of skull base. $^{7}$ The orbito-meningeal band is at the junction of basi-frontal and temporal lobe and is the lateral aspect of superior orbital fissure.

Once the $\mathrm{OMB}$ is dissected under high magnification (Figure 2), one can clearly observe the flattening of the cerebral gyri, providing a plane 

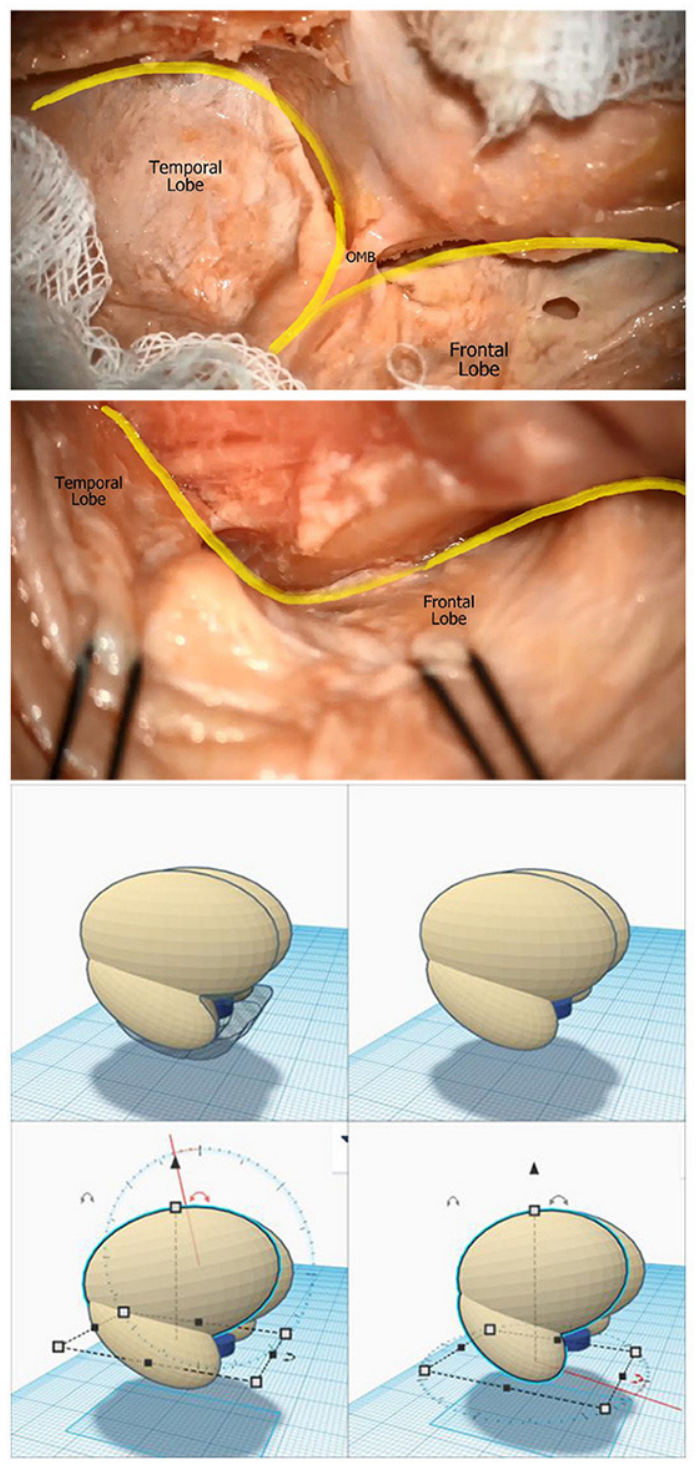

Figure 1. Before (a) and after (b) unlocking the brain in axial plane. One can clearly see how the brain folds are opened and the brain flattens out allowing exposure of the clinoidal and cavernous regions. (c) A graphical representation of the unlocking technique in axial and sagittal planes

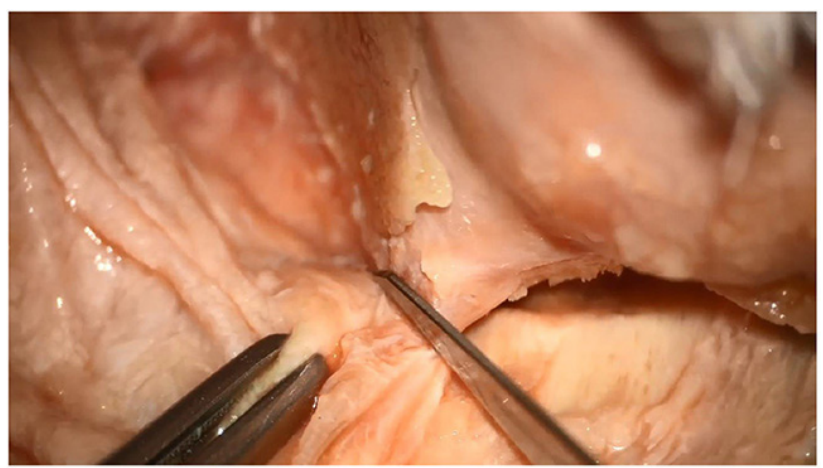

Figure 2. Sharply dissecting the orbito-meningeal band at a high magnification

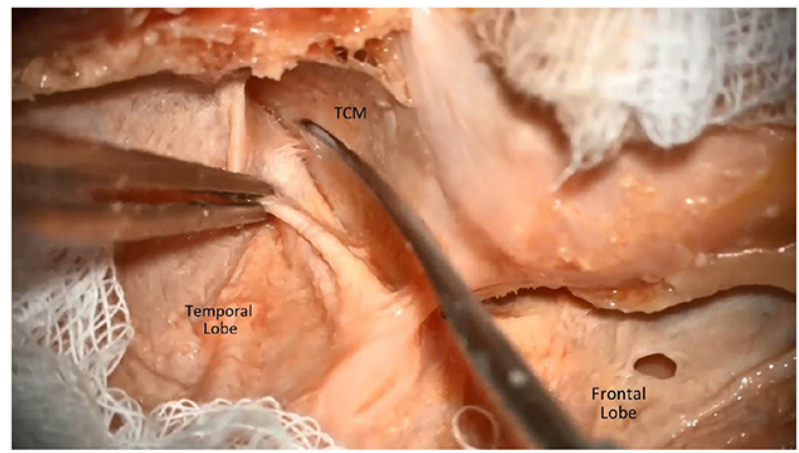

Figure 3 (a). Dissecting off or "peeling" the temporal dura from the cavernous sinus ensuring a pericavernous plane.

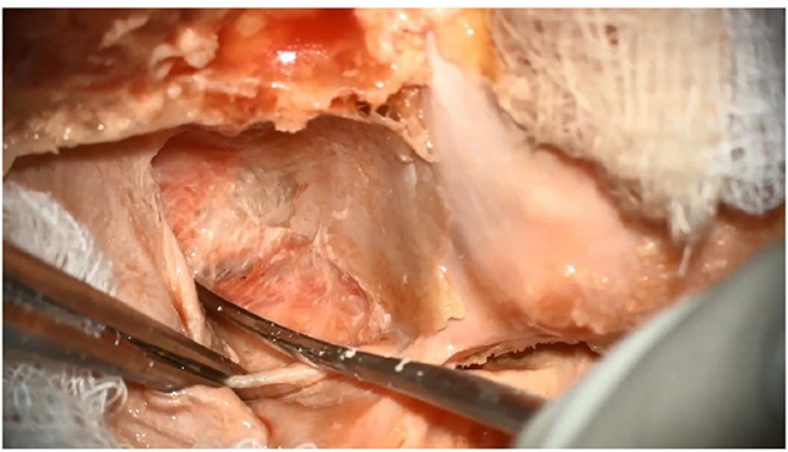

Figure 3 (b). Continuous peeling of the temporal dura from the cavernous sinus. The true cavernous membrane remains intact to ensure a bloodless dissection.

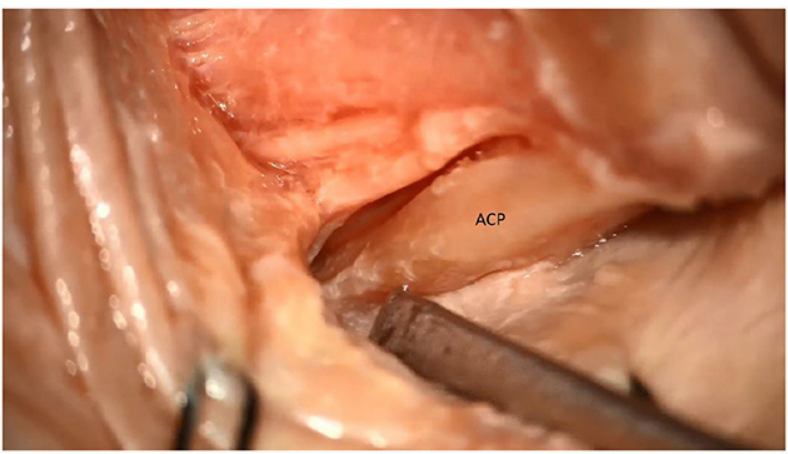

Figure $4(\mathrm{a})$. Uncovering the anterior clinoid after OMB dissection

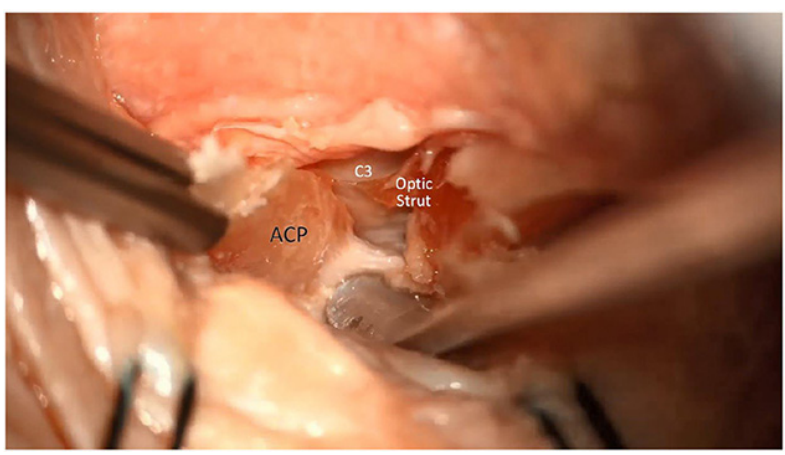

Figure 4 (b). Removing the Anterior Clinoid Process and the optic strut to visualize the optico-carotid window 
where the frontal lobe dura can be dissected off the anterior clinoid and the temporal dura can be dissected off the true cavernous membrane of the cavernous sinus. This uncovers the clinoid for easier anterior clinoidectomy and displaces the temporal lobe laterally in an extradural fashion, thus enabling axial unlocking and pericavernous peeling. ${ }^{2}$

\section{MODIFIED DOLENC APPROACH - AN APPROACH TO BLOODLESS EXTRADURAL PERICAVERNOUS DISSECTION:}

Sharp dissection of the OMB allows to view the true cavernous membrane. This membrane hosts the neurovascular structures of the cavernous sinuses, hence making it an important structure when dissecting in the region. Upon reaching true cavernous membrane plane, a sharp dissection can be performed in a peri-cavernous plane (Figure $3(\mathrm{a}))$. At the level of $\mathrm{V} 2$ and below, this plane is absent, but the interdural plane may be maintained, thereby decreasing the bleeding. This approach is a modification of the traditional Dolenc, ${ }^{3}$ as it ensures the intact TCM throughout the pericavernous dissection, preventing the bleeding that otherwise occurs in the Dolenc approach. If the bleeding occurs however, fibrin glue and surgicel can be used to overcome it. ${ }^{5,4}$ However care must be taken with the injection volume of fibrin as it may cause change in the venous drainage pattern with subsequent venous congestion. ${ }^{8}$

Using this modification of the Dolenc, the dissection is extended behind V3, and the middle meningeal artery is coagulated and divided, the GSPN can be seen as a dural band rather than a nerve. This "peeling" of the temporal dura in a trans-cavernous plane ensures that the nerves of the cavernous sinuses remain covered by the TCM (Figure 3(b)).

\section{ANTERIOR CLINOIDECTOMY}

The dissection of the OMB exposes the anterior clinoid process that is covered laterally by the frontal and temporal dural folds (Figure 4(a)). The basi-frontal dura can be sharply dissected off the anterior clinoid to completely uncover it. This makes anterior clinoidectomy easier and safe.

Orbital roof removal to uncover the extradural optic nerve can also be combined along with the dural cutting and sectioning the distal dural band. This allows mobilization of the carotid and increase space while dealing with aneurysms of this region. ${ }^{6}$

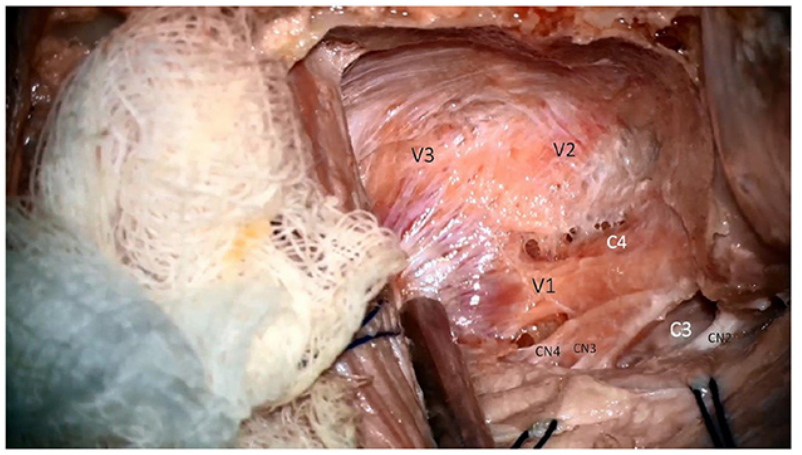

Figure 5. The Cavernous sinus after trans-cavernous peeling of the temporal dura.

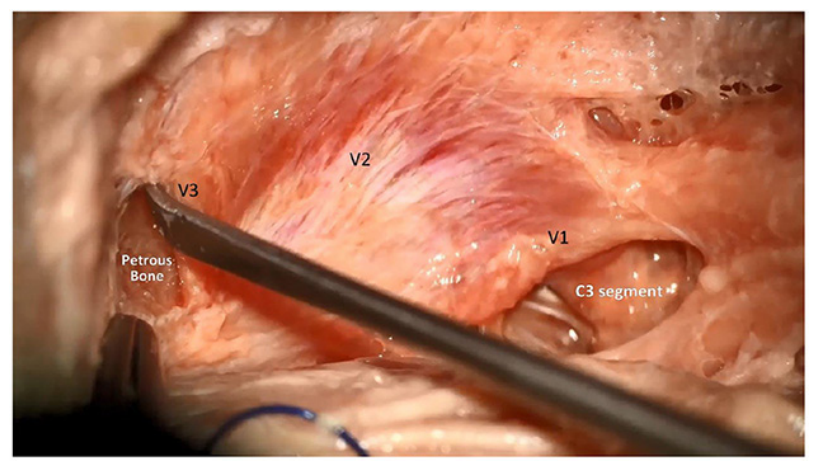

Figure 6. Approaching the petrous bone and mobilizing the V3

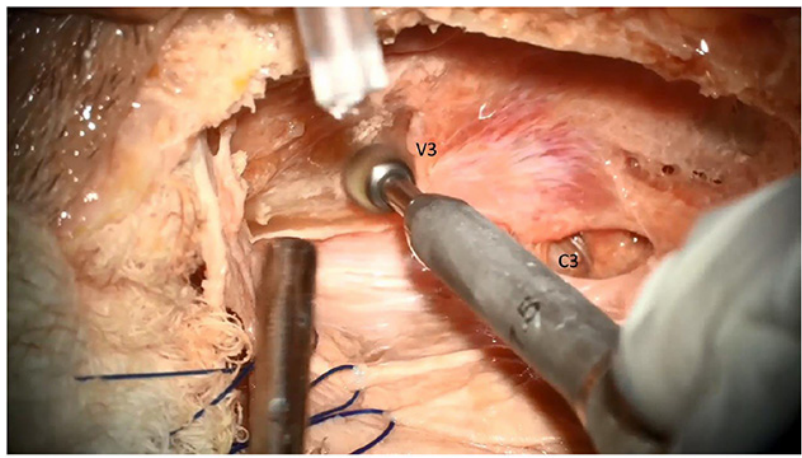

Figure 7. Drilling of the V3 to allow mobilization of the Gasserian Ganglion

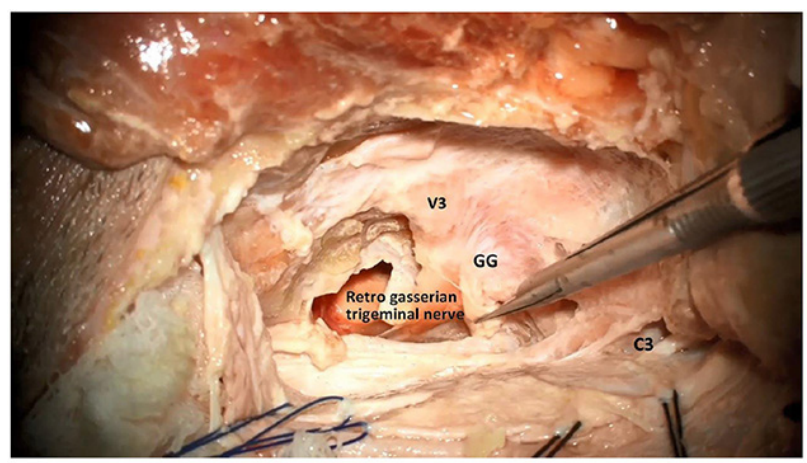

Figure 8. Continued dissection of the petrous fossa. [GG: Gasserian Ganglion] 
By complete removal of the ACP followed by careful extraction of the optic strut, one can visualize both the extra and intra dural boundaries of the optico-carotid window and reach the inter-optic or the suprasellar cisterns (Figure 4(b)). This is where the entire skull base is centered, and this approach can allow for access to all sellar and supra-sellar lesions. A panoramic view of the cavernous sinus

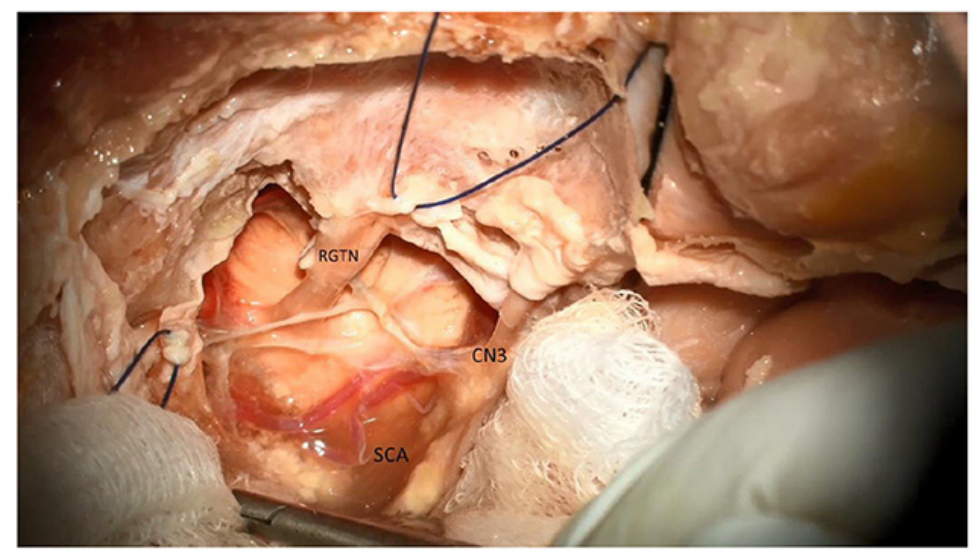

Figure 9. Exposing the lateral Pons up to the Occulomotor Nerve (CN3)
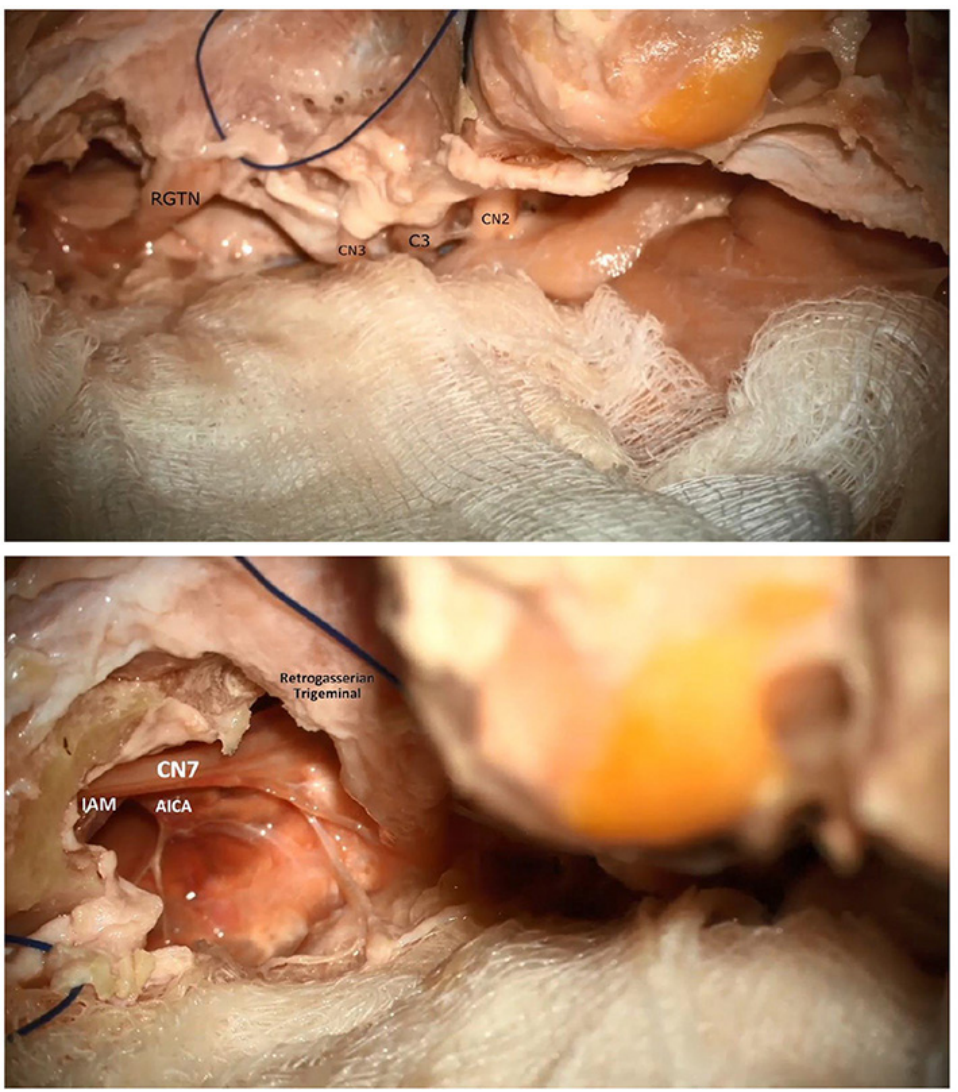

Figure 10 (a) (b): Using a combined middle fossa and modified Dolenc approach, one can expose the cranial nerves from $\mathrm{CN} 2$ to $\mathrm{CN} 7$ can be achieved after peeling the temporal dura (Figure 5). Complete unlocking of the frontal lobe from the temporal lobe is achieved after removal of the anterior clinoid process, and in some cases, followed by a posterior clinoidectomy. ${ }^{1}$ However, such a procedure should be reserved for complex cases and needs surgical expertise.

\section{PETROUS ANATOMY AND ANTERIOR PETROSECTOMY}

Once the dissection behind V3 is carried out, one must identify GSPN as a dural band thus protecting the nerve in the plane below. The middle meningeal artery is coagulated and cut to attain this plane[v]. Continuous dissection will take one to the petrous ridges and the arcuate eminence may be identified (Figure 6).

The V3 may be drilled into the infratemporal fossa and this will help in mobilizing the Gasserian ganglion anteriorly so that one could drill more of the petrous apex (Figure 7).

Posteriorly, one can unroof the internal auditory meatus and even do some drilling posterior to Internal auditory meatus. As one removes the petrous bone, the petrous carotid is found in the lateral boundary and the cochlea which is anterolateral to IAM is avoided.

The posterior fossa dura is exposed till one reaches the inferior petrosal sinus and the jugular tubercle is visualized (Figure 8). Dural opening is a combination of temporal and posterior fossa durotomies after which the superior petrosal sinus is bipolarized off and the tent is cut to the incisura posterior to the entrance of the 4 th nerve (Figure 9).

\section{ACKNOWLEDGMENTS}

None.

Conflict of Interest There is no conflict of interest related to the materials, methods, and findings in this study.

\section{FUNDING}

None.

\section{AUTHORS' CONTRIBUTIONS}

Authors took part in design of the study, contributed to data collection, participated in writing the manuscript and all agree to accept equal responsibility for accuracy of the contents of this article. 


\section{REFERENCES}

1. Cherian I, Kasper EM, Agarwal A. Technique of posterior clinoidectomy and its applications. Asian J Neurosurg 2018;13:777-8 DOI: 10.4103/ajns.AJNS_200_16

2. Cherian I, Kasper EM, Agarwal A. The orbitomeningeal band as a way to bloodless transcavernous dissection and anterior clinoidectomy. Asian J Neurosurg 2018;13:943-5. DOI: 10.4103/ajns.AJNS_198_16

3. Dolenc VV: Anatomy and Surgery of the Cavernous Sinus. Vienna, SpringerVerlag, 1989, pp 3-137.

4. Kassam A, Nemoto E, Balzer J, Rao G, Welch WC, Kuwabara $\mathrm{H}$ et al. Effects of Tisseel fibrin glue on the central nervous system of nonhuman primates. Ear, nose, \& throat journal. $2004 \mathrm{Apr} ; 83(4): 246-256$

5. Krayenbühl N1, Hafez A, Hernesniemi JA, Krisht AF. Taming the cavernous sinus: technique of hemostasis using fibrin glue. Neurosurgery. 2007 Sep;61(3 Suppl):E52; discussion E52. https://doi.org/10.1227/01. neu.0000289712.72555.9c
6. Mishra, S., Leão, B., \& Rosito, D. M. (2017). Extradural anterior clinoidectomy: Technical nuances from a learner's perspective. Asian Journal of Neurosurgery, 12(2), 189193. http://doi.org/10.4103/1793-5482.145544 PMID: 28484528

7. Nathal E, Gomez-Amador JL. Anatomic and surgical basis of the sphenoid ridge keyhole approach for cerebral aneurysms. Neurosurgery. 2005 Jan;56(1 Suppl):178-85; discussion 178-85. PMID: 15799808

8. Toyooka T. et al. Effect of Fibrin Glue Injection Into the Cavernous Sinus for Hemostasis During Transcavernous Surgery on the Cerebral Venous Draining System. Oper Neurosurg (Hagerstown). 2017 Apr 1;13(2):224-231. doi: 10.1227/NEU.0000000000001324

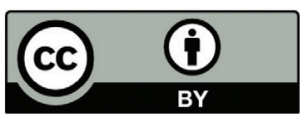

This work is licensed under a Creative Commons Attribution 